\title{
Inovação, propriedade intelectual e acesso a medicamentos e vacinas: 0 debate internacional na pandemia da COVID-19
}

\author{
Innovation, intellectual property and access to medicines and \\ vaccines: the international debate in the COVID-19 pandemic
}

\begin{abstract}
Claudia Chamas*
RESUMO: Este estudo analisa o debate internacional sobre vários aspectos do acesso a produtos de saúde, notadamente medicamentos e vacinas, no âmbito da Covid-19: estratégias de inovação (por exemplo, reposicionamento de fármacos), a discussão pública de alto nível sobre vacinas como bens públicos globais, os direitos de propriedade intelectual e as flexibilidades do Acordo TRIPS, os acordos para transferência de tecnologia e as questões de comércio. A pandemia concentrou imensos esforços tecnológicos, que, no futuro, podem estimular a revitalização das políticas públicas de ciência, a inovação e produção, as competências estatais, as parcerias público-privadas, e a alocação estratégica de investimentos contribuindo para os processos de catching-up tecnológico.
\end{abstract}

Palavras-chave: Propriedade Intelectual; Patente; Saúde; COVID-19; Coronavírus.

ABSTRACT: This study analyses the international debate on various aspects access to health products, particularly medicines and vaccines, for Covid-19: innovation strategies such as drug repurposing, highlevel public debate about vaccines as global public goods, intellectual property rights and TRIPS flexibilities, technology transfer agreements, and trade issues. The pandemic has concentrated intense technological efforts, which, in the future, may stimulate renewed public policies on science, innovation and production, state capabilities, public-private partnerships, and the strategic allocation of investments contributing to technological catching-up processes.

Keywords: Intellectual Property; Patent; Health; COVID-19; Coronavirus.

\footnotetext{
a Centro de Desenvolvimento Tecnológico em Saúde, Fundação Oswaldo Cruz, Instituto Nacional de Ciência e Tecnologia de Inovação em Doenças de Populações Negligenciadas, Rio de Janeiro, RJ, Brasil.

*Correspondência para/Correspondence to: Claudia Chamas. E-mail: claudia.chamas@cdts.fiocruz.br. Endereço: Avenida Brasil, 4036, sala 814, CEP 21040-361.

Recebido em/Received: 15/08/2020; Aprovado em/Approved: 06/11/2020.

Artigo publicado em acesso aberto sob licença $\mathrm{CC}$ BY 4.0 Internacional $@(i)$
} 


\section{INTRODUÇÃO}

Em setembro de 2019, o Global Preparedness Monitoring Board (GPMB)', iniciativa de avaliação independente para assegurar o cuidado adequado em crises globais de saúde, publicou o relatório $A$ World at Risk. $O$ estudo apontou intervenções urgentes para viabilizar preparações para emergências, que podem ser provocadas por diversos fatores, tais como: o crescimento populacional, a intensa urbanização, as economias interconectadas, o aumento das viagens, as situações de conflito e o desequilíbrio ambiental (WHO, 2019).

A análise menciona que, no período entre 2011 e 2018, a Organização Mundial de Saúde (OMS) detectou 1.483 eventos epidêmicos em 172 países. Em face das numerosas infecções emergentes e reemergentes (Zika, Ebola, Middle East Respiratory Syndrome, $\mathrm{H} 1 \mathrm{~N} 1$, etc), o grupo defendeu que, em casos de calamidades sanitárias, as ações para preparar estratégias, detectar ocorrências e oferecer respostas devem ser bens públicos globais (WHO, 2019).

Em 31 de dezembro de 2019 o escritório chinês da OMS recebeu notificação de casos de pneumonia de etiologia desconhecida em Wuhan, cidade mais populosa da China central com cerca de 11 milhões de habitantes. Em 7 de janeiro de 2020 cientistas chineses isolaram um novo coronavírus ( $\mathrm{CoV}$ ), cuja identificação da sequência genética desse vírus, acessível de modo aberto na base de dados da Global Initiative on Sharing All Influenza Data, permitiu o desenvolvimento de diagnóstico por meio de RT-PCR ${ }^{2}$ (WANG et al, 2020). Ainda está sob investigação dos pesquisadores o momento em que o novo vírus, que recebeu a recebeu a identificação Severe Acute Respiratory Syndrome Coronavirus $^{2}$ (SARS-CoV-2) ${ }^{3}$, saltou de animais para humanos e se manifestou como COVID-19 - como a doença passou a ser identificada (WHO, 2020a).

Com base na rápida multiplicação dos casos na China e no espalhamento em inúmeros países, em 30 de janeiro de 2020, a OMS caracterizou a situação como uma Public Health Emergency of International Concern, em atendimento ao Regulamento Sanitário Internacional, de 2005 (WHO, 2020b). Em 11 de março de 2020 a OMS declarou a ocorrência de uma pandemia - a primeira no mundo causada por um coronavírus (WHO, 2020c).

A busca por medicamentos e vacinas é o objetivo mais aguardado, o que gerou uma corrida acelerada de empresas, universidades e institutos de pesquisa em busca de produtos eficazes e seguros. Historicamente, as políticas de acesso a terapias têm sido discutidas de maneira exaustiva em vários fóruns internacionais, sem que, contudo, haja soluções satisfatórias, em especial para as populações negligenciadas. Parte substancial da população global não tem acesso aos medicamentos mais básicos, levando ao sofrimento, à pobreza extrema e a milhares de mortes evitáveis. Acrescida a essa situação crônica, a pandemia da Covid-19 fez emergir novos desafios para o acesso a produtos de saúde e acentuou as condições de desigualdade.

A potência da crise sanitária carrega um conjunto de elementos complexos com obstáculos para saídas equitativas - governança global sem consensos, crise do

\footnotetext{
1 O GPMB foi lançado em 2018 em resposta à UN Secretary General's Global Health Crises Task Force e convocado pela OMS e pelo Banco Mundial, sob a liderança de Gro Harlem Brundtland, ex-primeira ministra da Noruega e ex-diretorageral da OMS, e Elhadj As Sy, presidente do Conselho da Fundação Kofi Annan e ex-secretário geral da International Federation of Red Cross and Red Crescent Societies.

${ }^{2}$ Transcrição reversa e reação em cadeia da polimerase (em inglês, reverse transcription polymerase chain reaction).

3 Inicialmente denominado 2019-novel coronavirus (2019-nCoV).
} 
multilateralismo, disputas entre os Estados Unidos e a China, tensões políticas que envolvem a Organização Mundial de Saúde, crescentes expedientes bilaterais, incertezas inerentes à produção tecnológica acelerada.

No escopo da pandemia, um dos itens críticos é a capacidade de inovar com rapidez e difundir os resultados do desenvolvimento para todos os países necessitados. Aqui aflora o debate sobre a proteção intelectual - nesse contexto, patentes são instrumentos de incentivo ou barreiras ao acesso justo? Qual o papel do know how e da transferência de tecnologia? Alguns países rapidamente se mobilizaram para alterar o seu ordenamento jurídico, aperfeiçoando os instrumentos de licença compulsória. No plano internacional, destaca-se a ênfase no enquadramento das vacinas como bens públicos globais, propostas de pool de patentes e a formação de coalizões pautadas pelo caráter humanitário, mas também outras vias, como a compra antecipada de lotes de imunizantes e os diversos acordos comerciais entre governo e empresa.

Este artigo identifica e analisa a evolução do debate internacional sobre os diversos aspectos do acesso a produtos de saúde no âmbito da Covid-19, notadamente medicamentos e vacinas, bem como as relações com os direitos de propriedade intelectual, a transferência de tecnologia e as demandas de comércio. Quanto ao método, trata-se de um estudo exploratório, de natureza retrospectiva. Foram utilizados dados secundários de organizações governamentais e não-governamentais, literatura especializada, normas e mídia. Há limitações: o tema sofrerá mudanças inevitáveis, especialmente quando houver desfechos para os ensaios clínicos das vacinas ora em curso. A despeito de eventuais alterações nos posicionamentos de organismos internacionais e dos sistemas de saúde dos países quanto às recomendações de vacinas e os melhores caminhos para o acesso, os mapeamentos no corrente estágio de desenvolvimento são relevantes, posto que auxiliam a compreensão de um tema árido e com muitas variáveis, e podem ser úteis como subsídios à formulação de políticas públicas.

O artigo está estruturado da seguinte maneira: esta introdução caracteriza o cenário e descreve o objetivo e o método, a primeira parte revela como a pandemia trouxe novos desafios à inovação em medicamentos e vacinas. Em seguida, examina-se a reorganização do sistema patentário e outras questões de comércio em face da demanda sanitária, com relevo para as iniciativas de licenças compulsórias e pool de patentes; explora-se o debate de alto nível sobre os obstáculos ao acesso global a vacinas; e investiga-se a via de negociação direta entre países e empresas para o abastecimento das populações. Por último, apresentam-se algumas lições preliminares da pandemia de Covid-19.

\section{INOVAÇÃO EM MEDICAMENTOS E VACINAS NA PANDEMIA DA COVID-19}

O advento da Covid-19 trouxe um conjunto de desafios para a ciência - compreensão das características do vírus e da interação com o ser humano, entendimento dos aspectos sistêmicos relacionados à doença, imunidade, melhores tratamentos para os infectados e formas de prevenção. O enfrentamento da doença, com a precisão e celeridade esperadas pela população mundial, exigiu mobilizar inúmeras estruturas já consolidadas de pesquisa, inovação e produção, nos setores público e privado. As respostas até aqui oferecidas não seriam possíveis sem a cumulatividade tecnológica adquirida por meio de investimentos constantes e volumosos, políticas de apoio à pesquisa e ao desenvolvimento, aprendizados prévios sobre a família coronavírus, formação de pessoal, estruturas regulatórias, rede capacitada de negociação de 
tecnologias, capacidade de gerar inovações incrementais, inclusive na adaptação de estruturas produtivas.

Inicialmente, vale lembrar, de modo breve, algumas características da inovação na indústria farmacêutica. $O$ setor de medicamentos e vacinas é fortemente baseado em ciência. Uma fonte importante de conhecimento para as empresas privadas é a ciência produzida em universidades e institutos públicos (ORSENIGO et al, 2006). A via da imitação, estratégias de inventing around ou combinatórias, melhoramento contínuo das inovações existentes constituem maneiras usuais de aprendizado tecnológico e competitividade do setor. Entretanto, o lançamento de inovações na forma de novas entidades moleculares (New Chemical Entities) com demonstração de novas propriedades terapêuticas não é um evento tão frequente (BOTTAZZI et al, 2001).

Grosso modo, as inovações derivam de ações de longo prazo com riscos e incertezas elevados, investimentos robustos e estratégias bem definidas. As firmas acumulam capacidades de inovação com vista à obtenção de lucros extraordinários e ao distanciamento da concorrência (PARANHOS et al, 2020). As principais estruturas de pesquisa e desenvolvimento estão fortemente concentradas em países desenvolvidos. Historicamente, nos países menos favorecidos, a indústria dedicava-se às etapas de finalização, distribuição e/ou comercialização de medicamentos (CHAMAS e REIS, 2018; GADELHA et al, 2003; HASENCLEVER et al, 2008).

Nas últimas décadas do século XX, nas economias emergentes, surgiram esforços de aprimoramento do desempenho técnico, desenvolvimento in house ou complexos processos de absorção de tecnologias - que não são triviais ou automáticos e envolvem elementos tácitos e explícitos ${ }^{4}$, favorecendo as acumulações tecnológicas e ampliando as capacidades inovadoras (DOSI, 1988).

Desde a década de 1980, a Índia investiu em políticas públicas visando aquisição de capacidades tecnológicas de adaptação e inovações incrementais por meio da engenharia reversa. Na década de 1990, o governo indiano lançou o Drugs and Pharmaceuticals Research Programme e optou por fazer uso de todo o período de carência do Acordo sobre Aspectos dos Direitos de Propriedade Intelectual relacionados ao Comércio (Acordo TRIPS), de 1994, maximizando os benefícios para a indústria da saúde (RAY, 2004).

O Brasil fez escolhas distintas com consequências para a estruturação do Complexo Industrial da Saúde. O país internalizou de imediato o Acordo TRIPS, dispensando o prazo de adaptação para a fase de proteção de produtos farmacêuticos e inclusive adotando dispositivos que oferecem proteção superior à exigida pelo acordo.

Ao fim da década, o cenário se transforma e uma série de medidas dá fôlego ao setor farmacêutico: investimentos na formação de pessoal - com o fortalecimento da pósgraduação, retomada da política industrial, mudanças no ordenamento jurídico voltadas ao estímulo à inovação e às parcerias, e aperfeiçoamento do quadro regulatório (PARANHOS et al, 2020).

O setor de vacinas é intensivo em capital e bastante exigente em conformidade regulatória para atender às exigências das autoridades sanitárias. Nos países de renda média, nomeadamente Índia, China e Brasil, há empresas e instituições públicas e

\footnotetext{
${ }^{4}$ Como explica FIGUEIREDO (2004), "a real transferência de tecnologia de economias industrializadas para economias em desenvolvimento envolve, de um lado, a gestão da aquisição, instalação e operação da tecnologia importada. De outro, implica assegurar o engajamento da organização recipiente em um contínuo e sistemático processo de aprendizagem tecnológica."
} 
privadas sólidas que aproveitaram as oportunidades do aumento das campanhas de imunização e expandiram seu parque produtivo (GORDON e SAMANT, 2018).

Desde o aparecimento da Covid-19, inúmeros grupos de pesquisa no mundo tentam contribuir com soluções, seja para o alívio imediato dos sintomas, seja retardando a evolução da doença, melhorando a sobrevida ou planejando intervenções de longo prazo. Uma das estratégias mais utilizadas tem sido o reposicionamento de fármacos (SENANAYAKE, 2020). A opção pela via da inovação incremental se justifica pela urgência que a pandemia impõe - iniciar buscas por novas moléculas exigiria muito tempo e recursos financeiros que nem sempre estão disponíveis. Resgatar substâncias ${ }^{5}$ que já demonstraram segurança para uso em humanos tem gerado uma ampla movimentação da ciência mundial e apresentado bons candidatos à redefinição de uso terapêutico. Aproveitamento para Covid-19 precisa ser confirmado em termos de eficácia por meio de testes clínicos com coleta e análise de evidências confiáveis. Além dos antivirais de amplo espectro, outras classes e subclasses como glicocorticoides para mitigar danos inflamatórios têm sido testados.

Nesse contexto, a gestão dos megatrials se apresenta como um caminho para delimitar tratamentos eficazes para o enfrentamento da Covid-19. Uma das experiências é a Randomized Evaluation of Covid-19 Therapy (RECOVERY), lançada em março de 2020 e conduzida pela Universidade de Oxford, que testa: (1) o esteroide dexametasona em dose baixa ${ }^{6},(2)$ o antibiótico azitromicina, (3) o anti-inflamatório tocilizumabe, (4) o plasma convalescente coletado de doadores que se recuperaram do COVID-19 e contém anticorpos contra o vírus SARS-CoV-2, (5) o composto 4-aminoquinolina hidroxicloroquina ${ }^{7}$ e (6) o tratamento antiviral lopinavir-ritonavir8.

Outro megatrial em andamento é o Solidarity, lançado pela OMS com diversos parceiros, que também visa investigar as melhores opções de tratamento para a Covid19. A OMS informa que "cerca de 5.500 pacientes foram recrutados em 21 países" para participar de ensaios clínicos, além de mediar a negociação com fornecedores de opções eficazes para favorecer a acessibilidade. No momento, estão sendo apreciados: (1) Remdesivir e (2) Lopinavir / Ritonavir com Interferon beta-1a 9 .

O remdesivir é um exemplo de antiviral de amplo espectro com potencial eficácia terapêutica para ebola, atualmente em teste para Covid-19. Os dados preliminares indicaram redução no tempo de recuperação de adultos hospitalizados (BEIGEL et al, 2020). Nos Estados Unidos, a U.S. Food and Drug Administration emitiu uma autorização

\footnotetext{
5 E testes de combinações de moléculas.

${ }^{6}$ Estudo randomizado já publicado com demonstração de redução das taxas de mortalidade em cerca de um terço dos pacientes de Covid-19 hospitalizados com complicações respiratórias graves (THE RECOVERY COLLABORATIVE GROUP, 2020).

7 Segundo declaração da iniciativa RECOVERY: "hydroxychloroquine does not reduce the risk of death among hospitalised patients with this new disease". [Acesso em 3 de agosto de 2020]. Disponível em: https://www.recoverytrial.net/results/hydroxychloroquine-results Posição semelhante à agência governamental americana The Food and Drug Administration. [Acesso em 3 de agosto de 2020]. Disponível em: https://www.fda.gov/drugs/drug-safety-and-availability/fda-cautions-against-use-hydroxychloroquine-or-chloroquinecovid-19-outside-hospital-setting-or
}

${ }^{8}$ Segundo declaração da iniciativa RECOVERY: "for patients hospitalised with COVID-19 and not on a ventilator, lopinavir-ritonavir is not an effective treatment". [Acesso em 3 de agosto de 2020]. Disponível em: https://www.recoverytrial.net/results/lopinavar-results

9 [Acesso em 3 de agosto de 2020]. Disponível em: https://www.who.int/emergencies/diseases/novel-coronavirus2019/global-research-on-novel-coronavirus-2019-ncov/solidarity-clinical-trial-for-covid-19-treatments 
de uso emergencial ${ }^{10}$. Na Europa, o medicamento recebeu uma autorização condicional para introdução no mercado, de acordo com a seguinte justificativa:"the medicine addresses an unmet medical need and the benefit of immediate availability outweighs the risk from less comprehensive data than normally required"11.

No Brasil, algumas iniciativas estão em curso. Pesquisadores da Fundação Oswaldo Cruz (Fiocruz) lideraram teste do medicamento atazanavir, que faz parte do tratamento de pessoas que vivem com HIV/Aids. A instituição já concluiu o processo de absorção tecnológica ${ }^{12}$ da molécula e executa a produção com regularidade. Trata-se de um aspecto importante para facilitar o acesso à terapia, caso os estudos clínicos confirmem a eficácia. No estudo in vitro, o atazanavir mostrou-se eficaz na inibição da replicação do novo coronavírus e redução da produção de citocinas ligadas ao processo inflamatório pulmonar ${ }^{13}$ (SACRAMENTO et al, 2020).

Há outros exemplos no contexto brasileiro, como o daclatasvir e outros, mas o propósito não é organizar uma lista exaustiva de candidatos à terapia. O mais relevante é destacar que a estratégia de reposicionamento de fármacos é uma oportunidade para a participação de grupos acadêmicos em países emergentes, uma vez que é um caminho promissor, menos complexo e um pouco mais rápido do que desenvolver um medicamento novo, mas ainda assim com riscos e incertezas inerentes ao desenvolvimento farmacêutico. Participar da rede mundial de busca de tratamentos eficazes ajuda a internalizar conhecimento no campo da Covid-19, ampliar a cooperação internacional e potencializar o acesso.

As vacinas são a esperada solução de longo prazo. A pandemia corrente evidenciou, mais do que todas as crises passadas, a necessidade de um modelo bem estruturado que permita desenvolver produtos seguros e eficazes em alta velocidade, assim como estudos clínicos adequados.

Como os medicamentos, as vacinas fazem parte da grande corrida tecnológica em busca de saídas para a Covid-19 e são essenciais para o controle da doença. A OMS avalia que existam 26 vacinas candidatas em avaliação clínica e 136 em avaliação préclínica ${ }^{14}$. Algumas usam plataformas de desenvolvimento clássicas, outras estão ancoradas em plataformas de nova geração ${ }^{15}$.

Duas das vacinas com desenvolvimento mais avançado são a de Oxford e a da Sinovac. Ambas fazem parte de acordos com entidades governamentais brasileiras, como será visto na próxima seção deste artigo. A vacina desenvolvida pela Universidade de Oxford (denominada ChAdOx1 nCoV-19 ou AZD 1222) e licenciada para a empresa multinacional AstraZeneca encontra-se em fase de testes clínicos randomizados ${ }^{16}$. $O$

\footnotetext{
${ }^{10}$ [Acesso em 21 de julho de 2020]. Disponível em: https://www.fda.gov/news-events/pressannouncements/coronavirus-covid-19-update-fda-issues-emergency-use-authorization-potential-covid-19-treatment

${ }^{11}$ [Acesso em 29 de julho de 2020]. Disponível em: https://www.ema.europa.eu/en/medicines/human/EPAR/veklury\#authorisation-details-section

${ }^{12}$ [Acesso em 3 de agosto de 2020]. Disponível em: https://www.far.fiocruz.br/2020/06/farmanguinhos-concluiabsorcao-tecnologica-do-atazanavir/

${ }^{13}$ [Acesso em 3 de agosto de 2020]. Disponível em: https://portal.fiocruz.br/noticia/fiocruz-investiga-acao-deantirretrovirais-contra-covid-19

${ }^{14}$ [Acesso em 6 de agosto de 2020]. Disponível em: https://www.who.int/publications/m/item/draft-landscape-ofcovid-19-candidate-vaccines

${ }^{15}$ Para uma boa revisão do assunto, veja (VAN RIEL e DE WIT, 2020).

${ }^{16}$ https://clinicaltrials.gov/ct2/show/NCT04400838
} 
grupo de Oxford se beneficiou de estudos anteriores feitos com outros coronavírus. A cumulatividade desse aprendizado específico trouxe vantagens frente aos concorrentes, especialmente em ambiente de expectativa de velocidade do desenvolvimento e ansiedade coletiva. Resultados preliminares favoráveis das fases 1 e 2 em relação à segurança, reatogenicidade e imunogenicidade deram impulso a uma série de arranjos contratuais com vista ao acesso a essa vacina, que é baseada em vetor de adenovírus de chimpanzé com expressão da proteína spike do SARS-CoV-2, provocando resposta do sistema imune em termos de anticorpos e células $T$ (FOLEGATTI et al, 2020). O uso dessa plataforma é um provável fator acelerador em relação a plataformas mais antigas, que usam o próprio patógeno e exigem avaliação de segurança mais demorada, além de facilidades para a produção.

Na lista de vacinas em teste, há uma variedade de plataformas (vacinas de RNA, DNA, SARS-CoV-2 inativado, e vírus atenuado, proteínas, entre outras), que podem influenciar na velocidade de acesso. Os ensaios da fase 3 estão em curso em alguns países com níveis altos de transmissão, entre eles a África do Sul e o Brasil, sob a liderança do Centro de Referência para Imunobiológicos Especiais da Universidade Federal de São Paulo (Crie/Unifesp) ${ }^{17}$ (MAHASE, 2020).

A vacina candidata da empresa Sinovac (vacina CoronaVac) usa vírus inativado para estimular a resposta imune e obteve resultados satisfatórios nas fases 1 e 2 do estudo randomizado para as questões de segurança e imunogenicidade, induzindo a formação de anticorpos neutralizantes ${ }^{18}$. A firma está em colaboração com o Instituto Butantan para a realização da fase 3, ora em andamento, após autorização da Agência Nacional de Vigilância Sanitária (Anvisa) ${ }^{19}$.

Enquanto as vacinas mais competitivas no momento estão em desenvolvimento na Europa, nos Estados Unidos e na China, há exemplos de pesquisas em países em desenvolvimento ${ }^{20}$, favorecendo a aprendizagem cumulativa na área de virologia e ampliando as oportunidades futuras de parceria.

\section{ACESSO, COMÉRCIO E PROPRIEDADE INTELECTUAL}

Além das frentes na seara tecnológica, o advento da Covid-19 gerou questões na interface entre o acesso, o comércio e a propriedade intelectual. $O$ enquadramento da gestão de medicamentos e vacinas como bens públicos globais ou por intermédio de soluções típicas de mercado movimentou os fóruns internacionais.

Uma das primeiras respostas foi uma série de operações com vista ao ajustamento do ordenamento jurídico de alguns países para aperfeiçoar ou implementar medidas de

\footnotetext{
https://www.clinicaltrialsregister.eu/ctr-search/trial/2020-001228-32/GB

17 Com autorização da Anvisa. [Acesso em 6 de agosto de 2020]. Disponível em: https://www.unifesp.br/campus/sao/hidden/coronavirus/1383-unifesp-participara-de-teste-de-vacina-para-covid-19desenvolvida-por-oxford

${ }^{18}$ [Acesso em 6 de agosto de 2020]. Disponível em: http://www.sinovac.com/?optionid=754\&auto_id=904

${ }^{19}$ [Acesso em 6 de agosto de 2020]. Disponível em: http://www.butantan.gov.br/noticias/anvisa-autoriza-testes-davacina-contra-o-coronavirus--voluntarios-serao-profissionais-de-saude

${ }^{20}$ Vacina indiana Covaxin desenvolvida pela empresa Bharat Biotech. [Acesso em 6 de agosto de 2020]. Disponível em: https://www.bharatbiotech.com/covaxin.html

Vacina contra o novo coronavírus com utilização de vírus influenza como vetor vacinal. [Acesso em 6 de agosto de 2020]. Disponível em: http://www.cpqrr.fiocruz.br/pg/pesquisadores-da-fiocruz-minas-estao-envolvidos-nodesenvolvimento-de-uma-vacina-contra-o-coronavirus/
} 
licenciamento compulsório e uso governamental. As motivações residiam nas incertezas relacionadas à produção tecnológica e eventuais preços abusivos e outras condições limitantes. Tais respostas preventivas somam-se às medidas jurídicas disponíveis localmente, ancoradas nas flexibilidades do Acordo TRIPS e na Declaração de Doha sobre TRIPS e Saúde Pública ${ }^{21}$, de 2001.

Podem-se citar: (1) Chile, em 17 de março de 2020: "Resolución $\mathrm{N}^{\circ}$ 896, solicitando el otorgamiento de licencias no voluntarias contempladas en el artículo $51 \mathrm{~N}^{\circ} 2$ de la ley $\mathrm{N}^{\circ} 19.030$ de Propiedad Industrial, para facilitar el acceso y disponibilidad a los medicamentos y tecnologías para la prevención y tratamiento del Coronavirus"22; (2) Equador: em 20 de março de 2020: "Resolución para requerir al Gobierno Nacional el establecimiento de licencias obligatorias y otras medidas que permitan garantizar el acceso gratuito y a costos asequibles de los productos farmacéuticos y tecnologías médicas"; (3) Austrália: em 27 de março de 2020: o Partido Trabalhista "called on the Government to explore using laws that allow for the exploitation of patents for government services in a crisis"23; (4) França, em 23 de março de 2020: Lei n²02029024, "Ordonner la réquisition de tous biens et services nécessaires à la lutte contre la catastrophe sanitaire" $\mathrm{e}$ "En tant que de besoin, prendre toute mesure permettant la mise à la disposition des patients de médicaments appropriés pour l'éradication de la catastrophe sanitaire"; (5) Canadá, em 25 de março de 2020: COVID-19 Emergency Response Act (the Emergency Response Act)," Part 12 amends the Patent Act to, among other things, provide that the Commissioner must, on the application of the Minister of Health, authorize the Government of Canada and any person specified in the application to make, construct, use and sell a patented invention to the extent necessary to respond to a public health emergency that is a matter of national concern." 25; e (6) Alemanha: em 27 de março de 2020: o Conselho Federal (Bundesrat) aprovou uma lei focada na proteção da população em face da pandemia (Gesetz zum Schutz der Bevölkerung bei einer epidemischen Lage von nationaler Tragweite)26, permitindo, de acordo com a Seção 5, tomar providências de uso de invenções na área da saúde "no interesse do bem-estar público ou no interesse da segurança federal" 27.

Além das iniciativas citadas, em 19 de março de 2020, Israel emitiu uma permissão de uso governamental ${ }^{28}$ em Covid-19 para o medicamento Kaletra (lopinavir/ritonavir), tradicionalmente usado no tratamento da infecção pelo vírus da imunodeficiência humana (HIV). A medida visava a permitir importação da versão genérica de países em que a patente já havia expirado. A proteção doméstica segue até 2024. Logo após o

\footnotetext{
${ }^{21}$ [Acesso em 14 de agosto de 2020]. Disponível em:

https://www.wto.org/english/thewto_e/minist_e/mino1_e/mindecl_trips_e.htm

22 [Acesso em 6 de agosto de 2020]. Disponível em:

https://www.camara.cl/legislacion/resoluciones/resolucion_documentos.aspx?prmld=6028

${ }^{23}$ [Acesso em 6 de agosto de 2020]. Disponível em: https://brendanoconnor.com.au/news/latest-news/governmentshould-approve-use-of-patents-as-essential-goods-in-short-supply/

24 [Acesso em 6 de agosto de 2020]. Disponível em:

https://www.legifrance.gouv.fr/affichTexte.do?cidTexte=JORFTEXTo00041746313\&categorieLien=id

${ }^{25}$ [Acesso em 6 de agosto de 2020]. Disponível em: https://laws-

lois.justice.gc.ca/eng/AnnualStatutes/2020_5/FullText.html

${ }^{26}$ [Acesso em 6 de agosto de 2020]. Disponível em:

https://www.bgbl.de/xaver/bgbl/start.xav?startbk=Bundesanzeiger_BGBl\&jumpTo=bgbl120s0587.pdf\#_bgbl_\%2F\%2 F*\%5B\%40attr_id\%3D\%27bgbl120s0587.pdf\%27\%5D__1596994856183

27 "im Interesse der öffentlichen Wohlfahrt oder im Interesse der Sicherheit des Bundes benutzt werden soll".

${ }^{28}$ [Acesso em 6 de agosto de 2020]. Disponível em: http://freepdfhosting.com/645a6a5b51.pdf
} 
anúncio, a porta-voz da empresa Abbvie, titular das patentes do Kaletra, declarou que seriam tomadas medidas para remover quaisquer barreiras ao fornecimento, incluindo aspectos de propriedade intelectual (SILVERMAN, 2020, WHO, WIPO e WTO, 2020). A despeito dos resultados científicos da combinação farmacêutica, como visto na seção anterior deste artigo, a política da empresa se mostrou alinhada às expectativas de cooperação e solidariedade em um contexto de dificuldades globais.

No Brasil, os Projetos de Lei $n^{\circ} 1320^{29}, n^{0} 1462^{30}, n^{\circ} 1184^{31}$ e $n^{\circ} 2858^{32}$, em análise no Congresso Nacional, propõem modificações na Lei $n^{\circ}$ 9279, de 1996 - Lei da Propriedade Industrial, para facilitar e acelerar a concessão de licença compulsória durante o período de crise, em conformidade com a legalidade constitucional e os compromissos assumidos pelo Brasil no plano internacional (BARBOSA, 2020). As iniciativas impulsionaram mobilização da sociedade civil, em especial do Grupo de Trabalho sobre Propriedade Intelectual da Rede Brasileira pela Integração dos Povos (GTPI/Rebrip).

A licença compulsória, o uso governamental e o crown use não são institutos novos em situações de crise sanitária. Trata-se de medidas utilizadas no justo equilíbrio entre as necessidades de acesso e os direitos dos titulares. Durante os anos 2000, houve emprego dos dispositivos para facultar o abastecimento de antirretrovirais e de outras classes de medicamentos (BERMUDEZ et al, 2016; CASSIER e CORREA, 2003). Os Estados Unidos aventaram sua utilização no manejo da crise do Anthrax (ABBOTT, 2008). O uso efetivo para a Covid-19 depende de muitos fatores, sobretudo da autorização de opções terapêuticas para Covid-19, das relações com as condições de patenteabilidade e da disponibilidade via fabrico local ou importação.

Grosso modo, a produção de um medicamento sob proteção patentária depende de autorização do titular ou licença compulsória. No caso do remdesivir, a empresa titular depositou pedidos em diversos países e recentemente, anunciou faixas de preço do produto: US\$3.120 para tratamento de cinco dias com seis frascos, e variações ${ }^{33}$. A empresa negociou licenças voluntárias não-exclusivas e livres de royalties com produtores de genéricos na Índia, no Paquistão e no Egito, objetivando transferência de tecnologia, scale up da produção e distribuição em 127 países menos favorecidos, além de alguns emergentes, a preços determinados pelos licenciados. Brasil, Argentina e México não estão na lista de países-alvo do acordo (GILEAD, 2020).

Outro aspecto complementar se refere à disseminação da informação patentária de importância para as decisões relacionadas à pandemia. A OMPI criou o mecanismo de busca PATENTSCOPE Covid-19 Index ${ }^{34}$. A base de dados MedsPal ${ }^{35}$, da organização Medicines Patent Pool (MPP), rastreia pedidos de patente, patentes e eventuais licenças e termos de compromisso. Tais projetos constituem monitoramentos valiosos

\footnotetext{
29 [Acesso em 6 de agosto de 2020]. Disponível em:

https://www.camara.leg.br/proposicoesWeb/fichadetramitacao?idProposicao=2242509

${ }^{30}$ https://www.camara.leg.br/proposicoesWeb/fichadetramitacao?idProposicao=2242787

${ }^{31}$ [Acesso em 6 de agosto de 2020]. Disponível em:

https://www.camara.leg.br/proposicoesWeb/fichadetramitacao?idProposicao $=2242271$

32 https://www.camara.leg.br/proposicoesWeb/fichadetramitacao?idProposicao=2253612

33 “For governments of developed countries, including the U.S., the price will be lower, at $\$ 390$ per vial or $\$ 2,340$ per course“. [Acesso em 2 de julho de 2020]. Disponível em: https://www.fiercepharma.com/marketing/gilead-pricescovid-19-drug-remdesivir-line-cost-analysis

34 [Acesso em 2 de julho de 2020]. Disponível em: https://patentscope.wipo.int/search/en/covid19.jsf

35 [Acesso em 2 de julho de 2020]. Disponível em: https://www.medspal.org/?page=1 e
} 
para países com recursos escassos. O Instituto Nacional da Propriedade Industrial (INPI) criou o Observatório Covid-19 e produz estudos como o panorama de pedidos de patente do remdesivir no Brasil. As análises são úteis e servem de subsídios para diversos atores do sistema de inovação.

No âmbito das Nações Unidas, destaca-se a resolução da Assembleia Geral International cooperation to ensure global access to medicines, vaccines and medical equipment to face COVID-19 A/RES/74/274 ${ }^{36}$, de 8 de abril de 2020 , que solicita ao Secretário-Geral abordagens para o acesso justo e equitativo de medicamentos e futuras vacinas, com ênfase às necessidades dos países em desenvolvimento. No entanto, o texto não menciona questões relacionadas ao comércio e à propriedade intelectual. Publicado em 20 de março de 2020, o relatório das Nações Unidas Shared Responsibility, Global Solidarity: Responding to the socio-economic impacts of COVID-19 chama a atenção para a necessidade de remoção dos obstáculos para que ocorra o livre acesso aos resultados de pesquisa, assegurando que as vacinas estejam acessíveis a todos (UNITED NATIONS, 2020).

Lançado em 24 de abril de 2020, o Access to COVID-19 Tools (ACT) Accelerator é um mecanismo de colaboração global, que visa a acelerar o desenvolvimento e produção equitativa de vacinas e outras tecnologias de saúde com base nos esforços de múltiplos atores - Bill \& Melinda Gates Foundation, Coalition for Epidemic Preparedness Innovations, Gavi, the Vaccine Alliance, The Global Fund, Unitaid, Wellcome Trust, OMS e o setor privado ${ }^{37}$. No campo das vacinas, em especial no escopo do Covax - eixo de vacinas do ACT Accelerator, os propósitos são fincados no princípio da solidariedade: acelerar o desenvolvimento de uma vacina eficaz para todos os países; facilitar a construção de capacidade produtiva e compra antecipada de suprimentos; e distribuir 2 bilhões de doses de maneira justa até o final de 2021. É um enfoque alinhado à Agenda 2030, aos Objetivos de Desenvolvimento Sustentável, e à Estratégia Globale Plano de Ação sobre Saúde Pública, Inovação e Propriedade Intelectual (WHO, 2020d).

Para a universalização das vacinas, estimam-se US\$100 bilhões necessários. No entanto, como ressaltou Tedros Adhanom, Diretor Geral da OMS, em pronunciamento no dia 10 de agosto de 2020, há um vast global gap entre os recursos necessários para a luta contra o novo coronavírus e o montante comprometido até o momento - apenas 10\% do necessário, infelizmente (NEBEHAY, 2020, WHO, 2020e).

A Reunião Ministerial sobre Comércio e Investimentos do G20, em 30 de março de 2020, defendeu a disponibilidade de produtos farmacêuticos essenciais a preços acessíveis e de modo ágil, com incremento de produção e proteção contra especulação $(G 20,2020)$.

Em 20 de abril de 2020 uma Declaração Conjunta do então Diretor-Geral da Organização Mundial do Comércio (OMC), Roberto Azevêdo, e do Diretor-Geral da OMS, enfatizou como fatores críticos para a acessibilidade equitativa: "ensuring open access to clinical test results, the sharing of relevant intellectual property rights, increasing manufacturing capacity, open and transparent procurement regimes, the elimination of tariffs on relevant health technologies, and trade facilitation measures to reduce costs and delays" (WTO, 2020a).

\footnotetext{
${ }^{36}$ [Acesso em 2 de julho de 2020]. Disponível em: https://undocs.org/en/A/RES/74/274

37 O mecanismo Gavi Advance Market Commitment for COVID-19 Vaccines (Gavi Covax AMC) assinou termo de compromisso com a AstraZeneca para garantir 300 milhões de doses da vacina.
} 
A Resolução WHA73.1 aprovada pela última Assembleia Mundial da Saúde, em maio de 2020, faz um chamamento para um acesso justo e universal de produtos de saúde de interesse no combate à Covid-19 e pela remoção de barreiras injustificadas, reconhecendo que a vacinação é um bem público global e levando em consideração as flexibilidades do Acordo TRIPS e a Declaração de Doha sobre TRIPS e Saúde Pública. Ademais, o documento exorta as organizações internacionais a estabelecer pool de patentes em base voluntária, porém sem oferecer direcionamento sobre como coordenar licenças não-obrigatórias com amplo acesso.

Em resposta à proposta do Governo da Costa Rica, a OMS, em 29 de maio de 2020, lançou a iniciativa Solidarity Call to Action and the COVID-19 Technology Access Pool (CTAP), endossada por 39 países e outras partes. Os principais elementos incluem: "divulgação pública de sequências e dados genéticos; transparência em torno da publicação de todos os resultados de ensaios clínicos; os governos e outros financiadores são estimulados a incluir cláusulas em acordos de financiamento com empresas farmacêuticas e outros inovadores sobre distribuição equitativa, acessibilidade e publicação de dados de ensaio; licenciamento de qualquer tratamento potencial, diagnóstico, vacina ou outra tecnologia de saúde para o Medicines Patent Pool - um órgão de saúde pública apoiado pelas Nações Unidas que trabalha para aumentar o acesso e facilitar o desenvolvimento de medicamentos que salvam vidas para países de renda média e baixa; e promoção de modelos de inovação aberta e transferência de tecnologia que aumentem a produção local e a capacidade de fornecimento" (WHO, 2020f). Nesse escopo, o Conselho do Medicines Patent Pool decidiu expandir temporariamente o mandato da organização para contribuir com a reação à pandemia e oferecer sua expertise em licenciamento à OMS, com apoio da Unitaid (UNITAID, 2020).

A reunião do Conselho de TRIPS da OMC, ocorrida em 30 de julho de 2020, abordou o mérito da propriedade intelectual e as respostas à pandemia. Como consequência de uma requisição da África do Sul, os países em desenvolvimento frisaram que as tecnologias devem estar disponíveis e a propriedade intelectual não pode ser uma barreira ao acesso. Além disso, salientaram que as flexibilidades de TRIPS constituem instrumento para o enfrentamento da situação (WTO, 2020b). A Declaração da África do Sul menciona dois aspectos de grande importância, bastante negligenciados: as barreiras de propriedade intelectual não se restringem a patentes, e as flexibilidades em áreas como segredos comerciais não são bem compreendidas. O governo sulafricano ressaltou que há limitações nos modelos de licenças ad hoc voluntárias, pois não conseguiriam resolver adequadamente os obstáculos de propriedade intelectual (SYAM, 2020).

Em síntese, não resta dúvida que, no plano multilateral, há uma sintonia de vontades no sentido do fortalecimento da cooperação entre os diversos atores visando o suprimento justo de medicamentos e vacinas, livre de quaisquer empecilhos ao objetivo de solidariedade global. Até o momento, contudo, as ações multilaterais estão subfinanciadas e distantes de conferir tangibilidade a esses nobres propósitos.

Alguns países se organizam para antecipar a compra de mihões de doses das vacinas mais promissoras. A operação Warp speed, que envolve diversos órgãos governamentais dos Estados Unidos ${ }^{38}$, direcionou bilhões de dólares para 0

${ }^{8}$ Department of Health and Human Services, Centers for Disease Control and Prevention (CDC), Food and Drug Administration (FDA), National Institutes of Health (NIH), Biomedical Advanced Research and Development Authority (BARDA), e Department of Defense (DoD). 
desenvolvimento, produção e distribuição de vacinas, medicamentos e diagnósticos para a Covid-19. Foram anunciados investimentos nas vacinas candidatas da Johnson \& Johnson's, Moderna, AstraZeneca Universidade de Oxford, Novavax, Pfizer, Sanofi e GlaxoSmithKline, entre outras. Espera-se iniciar o fornecimento de vacinas à população americana até janeiro de 2021.

A Comissão Europeia adota a estratégia de negociação com os produtores de vacinas candidatas mais promissoras e pagamentos adiantados (upfront costs) por doses e produção, uma estratégia que abriga riscos, uma vez que não há garantias até que se complete a fase 3 de cada vacina em teste. Segundo relatos, a região busca postergar uma parte dos pagamentos até conseguir definição dos testes clínicos (GUARASCIO e POLLINA, 2020).

A estratégia brasileira de acesso a vacinas abarca duas instituições centenárias do Sistema Único de Saúde (SUS com suas capacidades tecnológicas e produtivas - a Fiocruz e o Instituto Butantan. A expertise e o longo histórico em negociação de transferência de tecnologias favorecem a exploração otimizada de opções no cenário internacional e o desenvolvimento de acordos que garantam o repasse do conhecimento tácito e explícito e permitam internalizar a tecnologia no Brasil.

Em 31 de julho de 2020 a Fiocruz e a AstraZeneca assinaram memorando de entendimento com as bases para o futuro acordo de transferência de tecnologia e produção de 100 milhões de doses da vacina, caso os ensaios mencionados na seção anterior comprovem a eficácia e segurança do produto. O acordo será fundamentado na modalidade "encomenda tecnológica", prevista na lei $n^{\circ} 10.973$, de 2004, em conformidade com as negociações do Ministério da Saúde. Para viabilizar a finalização da vacina e a etapa produtiva até que haja internalização do conhecimento tecnológico, haverá envio do Ingrediente Farmacêutico Ativo (IFA) e atividades de transferência de tecnologia de formulação, que incluem o know how e aquisição e ajustes dos equipamentos. Esta transferência está prevista para ocorrer mais rapidamente do que os processos anteriores. A Fiocruz também manifestou disposição para colaborar, ofertando sua capacidade técnica, com os processos de scaling up de outros parceiros em nível internacional (FIOCRUZ, 2020a, FIOCRUZ, 2020b).

Em 11 de junho de 2020 o governo do Estado de São Paulo e o Instituto Butantan anunciaram parceria com a empresa farmacêutica chinesa Sinovac para a produção da vacina CoronaVac. Caso os resultados da fase 3 dos ensaios clínicos sejam favoráveis, haverá celebração de acordo de transferência de tecnologia para escalonamento industrial no Brasil, domínio tecnológico e, assim como no caso da vacina negociada pela Fiocruz, fornecimento gratuito ao Sistema Único de Saúde (BUTANTAN, 2020).

O sucesso de quaisquer das vacinas será um fato positivo para o complexo industrial da saúde brasileiro, pois o Brasil terá incorporado conhecimento científico e tecnológico significativo em curto espaço de tempo, além de ter ampliado as parcerias internacionais.

\section{LIÇÕES PRELIMINARES DA PANDEMIA}

A pandemia trouxe incertezas, mas também suscitou uma intensa mobilização tecnológica, que pode estimular a revitalização das políticas públicas de ciência, inovação e produção, as competências estatais, as parcerias público-privadas, e a 
alocação estratégica de investimentos, contribuindo com os processos de catching-up tecnológico.

No momento em que esse texto é finalizado, não é possível ter plena certeza de como o quadro tecnológico se consolidará. As opções de medicamentos ainda não estão estabilizadas, os testes clínicos das vacinas candidatas encontram-se em curso e as políticas públicas para o acesso podem experimentar alterações. Observam-se, contudo, posicionamentos importantes em prol de uma visão humanitária e grande entusiasmo de diversos atores para encontrar soluções de uso comum, como ficou evidenciado na Assembleia Mundial de Saúde.

Grosso modo, o acesso à vacina tem sido planejado em duas linhas: no âmbito multilateral via abordagem solidária, porém ainda subfinanciada, e em negociação direta com as empresas com emprego de elevados recursos financeiros e tecnológicos e disposição ao risco para assegurar acesso preferencial. Esta tem sido a via protagonista, até o presente, embora possa haver fatos novos até que se tenha clareza sobre a eficácia dos produtos em teste.

Como vimos na introdução deste trabalho, a proposta do relatório $A$ World at Risk apontava abordagem de bens públicos globais como resposta às ações de mitigação de emergências sanitárias. Nesse escopo, a crise da Covid-19 tem sido pedagógica e oferece elementos para que, na preparação de eventos futuros, os países se articulem e decidam como coordenar a inovação, o comércio e a propriedade intelectual, e eliminar as barreiras à transferência das tecnologias. O acesso equitativo global fundamental para reduzir a vulnerabilidade das populações negligenciadas - depende, sobretudo, do compartilhamento do conhecimento, da boa vontade e da ação política.

\section{AGRADECIMENTOS}

Agradeço à Marcia Barros e Vivian Oliveira pelas valiosas contribuições.

\section{REFERÊNCIAS}

ABBOTT, Frederick M. Compulsory licensing for public health needs: The TRIPS Agenda at the WTO after the Doha Declaration on Public Health". 2008. Disponível em: https://quno.org/resource/2002/2/compulsory-licensing-public-health-needs. Acesso em: 03 ago. 2020.

BARBOSA, Pedro M. N. Licenças compulsórias e a covid-19. Disponível em: https://www.migalhas.com.br/depeso/324597/licencas-compulsorias-e-a-covid-19. Acesso em: 03 ago. 2020.

BEIGEL, John H.; TOMASHEK, Kay M.; DODD, Lori E.; MEHTA, Aneesh K; ZINGMAN, Barry S.; KALIL, Andre C. Remdesivir for the treatment of Covid-19 - preliminary report. 2020. Disponível em: doi: 10.1056/NEJMoaz007764. Acesso em: 03 ago. 2020.

BERMUDEZ, Jorge; OLIVEIRA, Maria; CHAVES, Gabriela. Novos medicamentos: quem poderá pagará. Cadernos de Saúde Pública, v. 32, supl. 2:e00025215, 2016. Disponível em: https://www.scielosp.org/article/csp/2016.v32suppl2/e00025215/pt/. Acesso em: 03 ago. 2020. 
BOTTAZZII, Giulio et al. Innovation and corporate growth in the evolution of the drug industry. International Journal of Industrial Organization, v. 19, n. 7, p. 1161-1187, 2001. Disponível em:

https://www.sciencedirect.com/science/article/abs/pii/S0167718701000686. Acesso em: 21 jul. 2020.

BUTANTAN. Butantan e Governo de SP vão testar e produzir vacina inédita contra coronavírus, 2020. Disponível em: http://www.butantan.gov.br/noticias/butantan-egoverno-de-sp-vao-testar-e-produzir-vacina-inedita-contra-coronavirus. Acesso em: 21 jul. 2020.

CASSIER, Maurice; CORREA, Marilena. Patents, innovation and public health: brazilian public-sector laboratories' experience in copying AIDS drugs. Economics of AIDS and Access to HIV/AIDS Care in Developing Countries. 2020. Disponível em:

https://halshs.archives-ouvertes.fr/halshs-02162784. Acesso em: 21 jul. 2020.

CHAMAS, Claudia; REIS, Roberto. Saúde pública, patentes e exclusividade de dados de testes clínicos. In: BAIOCCHI, Enzo; SICHEL, Ricardo Luiz (org.). 20 anos da lei $n^{\circ}$ 9.279/1996: lei da propriedade industrial. Rio de Janeiro: Lumen Juris, 2018.

DOSI, Giovanni. The nature of the innovative process. In: DOSI, Giovanni et al. (Ed.). Technical change and economic theory. London: Pinter, 1988.

FIGUEIREDO, Paulo. Aprendizagem tecnológica e inovação industrial em economias emergentes: uma breve contribuição para o desenho e implementação de estudos empíricos e estratégias no Brasil. Revista Brasileira de Inovação, v. 3, n. 2, 323-361, 2004. Disponível em:

https://periodicos.sbu.unicamp.br/ojs/index.php/rbi/article/view/8648901. Acesso em: 21 jul. 2020.

FIOCRUZ. Fiocruz e AstraZeneca alinham detalhes para produção de vacina para Covid19. 2020a. Disponível em: https://portal.fiocruz.br/noticia/fiocruz-e-astrazenecaalinham-detalhes-para-producao-de-vacina-para-covid-19. Acesso em: 21 jul. 2020.

FIOCRUZ. Covid-19: medida provisória garante produção de vacina. 2020b. Disponível em: https://agencia.fiocruz.br/covid-19-medida-provisoria-garante-producao-de-vacina Acesso em: 21 jul. 2020.

FOLEGATTI, Pedro M. et al. Safety and immunogenicity of the ChAdOx1 nCoV-19 vaccine against SARS-CoV-2: a preliminary report of a phase $1 / 2$, single-blind, randomised controlled trial. Lancet, 2020. Disponível em: doi:10.1016/S01406736(20)31604-4. Acesso em: 21 jul. 2020.

GADELHA, Carlos; QUENTAL, Cristiane; FIALHO, Beatriz. Saúde e inovação: uma abordagem sistêmica das indústrias da saúde. Cadernos de Saúde Pública, v. 19, n. 1, p. 47-59, 2003. Disponível em: https://www.scielo.br/scielo.php?pid=S0102311X2003000100006\&script=sci_abstract\&tlng=pt

GILEAD. Voluntary licensing agreements for remdesivir. 2020. Disponível em: https://www.gilead.com/purpose/advancing-global-health/covid-19/voluntarylicensing-agreements-for-remdesivir. Acesso em: 21 jul. 2020. 
GORDON, Douglas; SAMANT, Vijay B. "The vaccine industry." Plotkin's Vaccines, n. 48, 2020. Disponível em: https://www.sciencedirect.com/book/9780323357616/plotkinsvaccines. Acesso em: 21 jul. 2020.

G20. G20 trade and investment ministerial statement. Disponível em: http://www.g20.utoronto.ca/2020/2020-g20-trade-0330.html. Acesso em: 21 jul. 2020.

GUARASCIO, Francesco; POLLINA, Elvira. EU talks with Pfizer, Sanofi, J\&J on COVID vaccines hit snags - sources. Reuters. 2020. Disponível em:

https://www.reuters.com/article/us-health-coronavirus-eu-vaccines-exclus/exclusiveeu-talks-with-pfizer-sanofi-ji-on-covid-vaccines-hit-snags-sources-idUSKCN24S1YQ. Acesso em: 21 jul. 2020.

HASENCLEVER, Lia; PARANHOS, Julia; PAIVA, Vitor. A extensão da propriedade intelectual através do sigilo do registro de medicamentos: empecilhos à política de medicamentos genéricos. Reciis, n. 2, p. 50-57, 2008. Disponível em: https://www.reciis.icict.fiocruz.br/index.php/reciis/article/view/848. Acesso em: 21 jul. 2020.

MAHASE, Elisabeth. Covid-19: Oxford team begins vaccine trials in Brazil and South Africa to determine efficacy. 2020. Disponível em: https://www.bmj.com/content/369/bmj.m2612. Acesso em: 21 jul. 2020.

NEBEHAY, Stephanie. WHO decries 'vast global gap' in funds needed to fight coronavirus. 2020. Disponível em: https://www.reuters.com/article/us-healthcoronavirus-who/who-decries-vast-global-gap-in-funds-needed-to-fight-coronavirusidUSKCN25614V. Acesso em: 21 jul. 2020.

ORSENIGO, Luigi; DOSI, Giovanni; MAZZUCATO, Mariana. The dynamics of knowledge accumulation, regulation and appropriability in the pharma-biotech sector: policy issues. In: DOSI, Giovanni; MAZZUCATO, Mariana. Knowledge Accumulation and Industry Evolution. Cambridge, UK: Cambridge University Press, 2006.

PARANHOS, Julia; MERCADANTE, Eduardo; HASENCLEVER, Lia. Os esforços inovativos das grandes empresas farmacêuticas no Brasil: o que mudou nas duas últimas décadas?. Revista Brasileira de Inovação, v. 19, p. e0200015, 2020. Disponível em: https://periodicos.sbu.unicamp.br/ojs/index.php/rbi/article/view/8655780. Acesso em: 21 jul. 2020.

RAY, Amit. The changing structure of R\&D incentives in India: the pharmaceutical sector. Science Technology and Society, v. 9, n. 2, p. 297-317, 2004. Disponível em: https://journals.sagepub.com/doi/abs/10.1177/097172180400900205. Acesso em: 21 jul. 2020.

SACRAMENTO Carolina Q. et al. The in vitro antiviral activity of the anti-hepatitis $C$ virus (HCV) drugs daclatasvir and sofosbuvir against SARS-CoV-2. 2020. Disponível em: https://doi.org/10.1101/2020.06.15.153411. Acesso em: 21 jul. 2020.

SENANAYAKE, Suranga L. Drug repurposing strategies for COVID-19. Future Drug Discov, v. 0, n. 0, 2020. Disponível em: doi:10.4155/fdd-2020-0010. Acesso em: 21 jul. 2020. 
SILVERMAN, Ed. AbbVie will allow generic copies of its HIV pill in Israel after the government approved a license. Stat, 2020. Disponível em:

https://www.statnews.com/pharmalot/2020/03/20/abbvie-israel-hiv-kaletracoronavirus-covid19/. Acesso em: 21 jul. 2020.

SYAM, Nirmalya. WTO TRIPS Council discusses national IP measures and TRIPS flexibilities in the context of COVID-19. 2020. Disponível em: https://us5.campaignarchive.com/?u=fagcf38799136b5660f367ba6\&id=184960eo8b. Acesso em: 05 jun. 2020.

THE RECOVERY COLLABORATIVE GROUP. Dexamethasone in hospitalized patients with Covid-19: preliminary report. 2020. Disponível em: DOI: 10.1056/NEJMoa2021436. Acesso em: 05 jun. 2020.

UNITAID. Medicines patent pool and unitaid respond to access efforts for COVID-19 treatments and technologies. Geneva: UNITAID, 2020. Disponível em: https://unitaid.org/news-blog/medicines-patent-pool-and-unitaid-respond-to-accessefforts-for-covid-19-treatments-and-technologies/\#en. Acesso em: 21 jul. 2020.

UNITED NATIONS. Shared responsibility, global solidarity: responding to the socioeconomic impacts of COVID-19. NY, 2020. Disponível em: https://data2.unhcr.org/en/documents/details/75741. Acesso em: 21 jul. 2020.

WANG, Chen et al. A novel coronavirus outbreak of global health concern. The Lancet, v. 395, n. 10223, 2020.

WHO. Global preparedness monitoring board: a world at risk: annual report on global preparedness for health emergencies. Geneva: WHO, 2019. Disponível em: https://apps.who.int/gpmb/annual_report.html. Acesso em: 05 jun. 2020.

WHO. Pneumonia of unknown cause - China. Geneva, WHO, 2020a. Disponível em: https://www.who.int/csr/don/05-january-2020-pneumonia-of-unkown-cause-china/en/. Acesso em: jun. 2020.

WHO. Statement on the second meeting of the International Health Regulations (2005) Emergency Committee regarding the outbreak of novel coronavirus (2019nCoV). Geneva, WHO, 2020b. Disponível em: https://www.who.int/newsroom/detail/30-01-2020-statement-on-the-second-meeting-of-the-international-healthregulations-(2005)-emergency-committee-regarding-the-outbreak-of-novelcoronavirus-(2019-ncov). Acesso em: 05 jun. 2020.

WHO. WHO Director-General's opening remarks at the media briefing on COVID-19. Geneva: WHO, 2020c. Disponível em: https://www.who.int/dg/speeches/detail/whodirector-general-s-opening-remarks-at-the-media-briefing-on-covid-19---11-march-2020. Acesso em: 20 jul. 2020.

WHO. COVAX: working for global equitable access to COVID-19 vaccines. Geneva: WHO, 2020d. Disponível em: https://www.who.int/initiatives/act-accelerator/covax. Acesso em: 12 ago. 2020.

WHO. WHO Director-General's opening remarks at the media briefing on COVID-19. Geneva: WHO, 2020e. Disponível em: https://www.who.int/dg/speeches/detail/who- 
director-general-s-opening-remarks-at-the-media-briefing-on-covid-19--10-august2020. Acesso em: 21 jul. 2020.

WHO. International community rallies to support open research and science to fight COVID-19. Geneva: WHO, 2020f. Disponível em: https://www.who.int/newsroom/detail/29-05-2020-international-community-rallies-to-support-open-researchand-science-to-fight-covid-19. Acesso em: 21 jul. 2020.

WHO; WIPO; WTO. Promoting access to medical technologies and innovation: intersections between public health, intellectual property and trade. Geneva: WHO, 2020. Disponível em: https://www.wipo.int/publications/en/details.jsp?id=4511. Acesso em: 21 jul. 2020.

WTO. Joint statement by WTO Director-General Roberto Azevêdo and WHO DirectorGeneral Tedros Adhanom Ghebreyesus. Geneva: WHO, 2020a. Disponível em: https://www.wto.org/english/news_e/news20_e/igo_14apr20_e.htm. Acesso em: 21 jul. 2020.

WTO. WTO members stress role of IP system in fighting COVID-19. Geneva: WHO 202ob. Disponível em: https://www.wto.org/english/news_e/news20_e/trip_30jul20_e.htm. Acesso em: 21 jul. 2020. 